\title{
Determination of the Total Width of the $\eta^{\prime}$ Meson
}

\author{
E. Czerwiński, ${ }^{1,2, *}$ P. Moskal, ${ }^{1,2}$ D. Grzonka, ${ }^{2}$ R. Czyżykiewicz, ${ }^{1}$ D. Gil, ${ }^{1}$ B. Kamys, ${ }^{1}$ A. Khoukaz, ${ }^{3}$ J. Klaja, ${ }^{1,2}$ \\ P. Klaja, ${ }^{1,2}$ W. Krzemień, ${ }^{1,2}$ W. Oelert, ${ }^{2}$ J. Ritman, ${ }^{2}$ T. Sefzick, ${ }^{2}$ M. Siemaszko, ${ }^{4}$ M. Silarski, ${ }^{1}$ J. Smyrski, ${ }^{1}$ \\ A. Täschner, ${ }^{3}$ M. Wolke, ${ }^{2,5}$ P. Wüstner, ${ }^{2}$ J. Zdebik, ${ }^{1}$ M. Zieliński, ${ }^{1}$ and W. Zipper ${ }^{4}$ \\ ${ }^{1}$ Institute of Physics, Jagiellonian University, PL-30-059 Cracow, Poland \\ ${ }^{2}$ Institute for Nuclear Physics and Jülich Center for Hadron Physics, Research Center Jülich, D-52425 Jülich, Germany \\ ${ }^{3}$ IKP, Westfälische Wilhelms-Universität, D-48149 Münster, Germany \\ ${ }^{4}$ Institute of Physics, University of Silesia, PL-40-007 Katowice, Poland \\ ${ }^{5}$ Department of Physics and Astronomy, Uppsala University, SE-751 20 Uppsala, Sweden
}

(Received 10 May 2010; published 17 September 2010)

\begin{abstract}
Taking advantage of both the low-emittance proton beam of the cooler synchrotron COSY and the high momentum precision of the COSY-11 detector system, the mass distribution of the $\eta^{\prime}$ meson was measured with a resolution of $0.33 \mathrm{MeV} / c^{2}$ (FWHM), improving the experimental mass resolution by almost an order of magnitude with respect to previous results. Based on the sample of more than 2300 reconstructed $p p \rightarrow p p \eta^{\prime}$ events, the total width of the $\eta^{\prime}$ meson was determined to be $\Gamma_{\eta^{\prime}}=0.226 \pm$ 0.017 (stat) \pm 0.014 (syst) $\mathrm{MeV} / c^{2}$.
\end{abstract}

DOI: 10.1103/PhysRevLett.105.122001

PACS numbers: 14.40.Be, 13.60.Le, 14.70.Dj

In this Letter, we report on the measurement of the mass distribution of the $\eta^{\prime}$ meson carried out with a resolution of a fraction of $\mathrm{MeV} / c^{2}$. This accuracy was obtained by using the low-emittance proton-beam of the cooler synchrotron COSY [1] and the high momentum resolution of the COSY-11 detector system [2,3], and it is nearly an order of magnitude more precise than previous results.

In the latest review by the Particle Data Group (PDG) [4], two values for the total width of the $\eta^{\prime}$ meson are given. One of these values, $(0.30 \pm 0.09) \mathrm{MeV} / c^{2}$, results from the average of two measurements [5,6], though only in one of these experiments was $\Gamma_{\eta^{\prime}}$ extracted directly based on the mass distribution [5]. The second value $(0.205 \pm 0.015) \mathrm{MeV} / c^{2}$, recommended by the PDG, is determined by the fit to altogether 51 measurements of partial widths, branching ratios, and combinations of particle widths obtained from integrated cross sections [4]. The result of the fit is strongly correlated with the value of the partial width $\Gamma\left(\eta^{\prime} \rightarrow \gamma \gamma\right)$, which causes serious difficulties when the total and the partial width have to be used at the same time, like, e.g., in studies of the gluonium content of the $\eta^{\prime}$ meson $[7,8]$.

The partial width of the $\eta^{\prime} \rightarrow \gamma \gamma$ channel can be extracted from the $e^{+} e^{-} \rightarrow e^{+} e^{-} \eta^{\prime}$ cross sections without knowledge of the $\Gamma_{\eta^{\prime}}$ [9-11], yet its derivation is modeldependent due to the need to incorporate a form factor which describes the spatial distribution of the electric charge in the $\eta^{\prime}$ meson. For the derivation of partial widths of all other decay channels, the knowledge of $\Gamma_{\eta^{\prime}}$ is mandatory. At present it is the inaccuracy of $\Gamma_{\eta^{\prime}}$ which limits investigations of many interesting physics issues, such as, for example, the quark mass difference $m_{d}-m_{u}[12,13]$, isospin breaking in QCD [12,14], or the box anomaly of QCD [15]. This is because the branching ratios of the $\eta^{\prime}$ meson decay channels are typically known with a relative precision of more than an order of magnitude better than the present accuracy with which $\Gamma_{\eta^{\prime}}$ is extracted [4].

The signal of the $\eta^{\prime}$ meson production observed in previous experiments $[5,6,16-21]$ with mass resolutions poorer than $\sim 1 \mathrm{MeV} / c^{2}$ do not a priori exclude the possibility that some structure in the mass distribution of the $\eta^{\prime}$ meson would be visible at higher precision. Extractions of the $\eta^{\prime}$ width $\left(\Gamma_{\eta^{\prime}}\right)$ were performed under the assumption that the $\eta^{\prime}$ meson is a single state. This, however, must not necessarily be the case $[22,23]$ if there is a significant glue contribution in the wave function of this meson [24]. The precision achieved with the COSY-11 facility enabled us for the first time to determine the mass spectrum of the $\eta^{\prime}$ meson with a resolution comparable to its total width of $\sim 0.2 \mathrm{MeV} / c^{2}$ [4].

The experiment, reported in this Letter, was performed in the Research Centre Jülich. The value of $\Gamma_{\eta^{\prime}}$ was established directly from the measurement of the mass distribution of the $\eta^{\prime}$ meson, produced via the $p p \rightarrow p p \eta^{\prime}$ reaction. The momentum of the COSY beam and the dedicated zero degree COSY-11 facility enabled the measurement at an excess energy of only a fraction of an $\mathrm{MeV}$ above the kinematic threshold for the $\eta^{\prime}$ meson production. This was the most decisive factor in minimizing uncertainties of the missing-mass determination, since at threshold the partial derivative of the missing mass with respect to the outgoing proton momentum tends to zero. In addition, close to threshold the signal-to-background ratio increases due to the more rapid reduction of the phase space for multimeson production than for the $\eta^{\prime}$.

In order to control systematic uncertainties, the measurement was carried out at five different beam momenta, which were nominally $3211,3213,3214,3218$, and 
$3224 \mathrm{MeV} / c$. The cooled beam of protons [25] circulated in the ring of the cooler synchrotron COSY through a stream of the hydrogen cluster target [26]. In the magnetic field of the COSY dipole, the final state protons from the $p p \rightarrow p p \eta^{\prime}$ reaction were bent more than the beam protons and were measured by means of the COSY-11 detector shown schematically in Fig. 1. The momentum vectors of the outgoing protons were reconstructed based on the bending of their trajectories in the magnetic field between the center of the reaction region and the tracks measured in the drift chambers ( $D 1$ and $D 2)$. In addition, the velocities of the two protons were determined from their time of flight measured between the scintillator detectors $S 1$ and $S 3$. The independent determination of momentum and velocity enables particle identification to be made via its invariant mass. Since the momentum is reconstructed more precisely than the velocity, after the identification, the energy of the particle is derived from its known mass and momentum. The standard technique for monitoring the beam momentum at the COSY accelerator is through the measurement of the frequency distribution of the circulating beam. Such a distribution can be transformed to the momentum coordinate by using the values of the accelerator settings [25]. As an example, a spectrum for the lowest beam energy used in the experiment is presented in the left corner of Fig. 1. The beam momentum distribution is smooth and its spread is equal to $2.5 \mathrm{MeV} / c$ (FWHM). However, due to the position of the COSY-11 target system in a bending section of the COSY ring in a dispersive region, the effective spread of the beam (the momentum

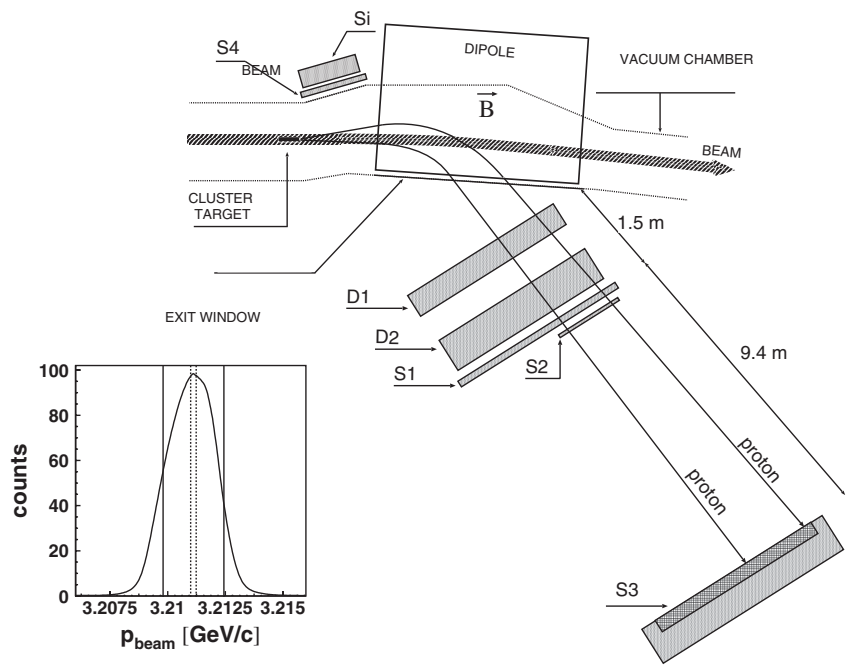

FIG. 1. Schematic view of the COSY-11 detector setup (top view). $S 1, S 2, S 3$, and $S 4$ denote scintillator detectors, $D 1$ and $D 2$ indicate drift chambers, and $\mathrm{Si}$ stands for the silicon-pad detector. Left corner: Momentum spectrum for the measurement with the nominal beam momentum of $3211 \mathrm{MeV} / c$. As an example, the effective spread of the beam momentum due to the dispersion is shown as for a target width of $1 \mathrm{~mm}$ (dashed line) and $1 \mathrm{~cm}$ (solid line). range seen by the target) is smaller [27]. The $\eta^{\prime}$ meson was not registered but instead it was identified by using the missing-mass technique. The precision of the determination of the size and position of the target stream influences the accuracy of the reconstruction of the momentum of the outgoing particles and the accuracy of the determination of the momentum spread of beam protons interacting with the target. As a compromise between accuracy and statistics, the transverse size of the target stream was reduced to $0.9 \mathrm{~mm}$, which is significantly less than the horizontal spread of the COSY beam. Therefore, the momentum spread of the interacting protons is defined by the momentum dispersion at the target region and by the size of the target stream. The size and position of the target stream, being crucial for the analysis, were monitored by two independent methods. The first was based on the measurement of the momentum distribution of elastically scattered protons [28], while the second was a direct measurement of the target geometry by mechanically scanning the target stream position above and below the target area from time to time. A diagnostic unit with several wires was rotated through the target stream, and the pressure in the cluster beam dump was measured as a function of the wire position. When parts of the target stream are blocked by a wire, the pressure decreases proportional to the blocked area. Therefore variations of the pressure allowed the monitoring of the size and alignment of the target stream during the experiment. The results of the two methods are in good agreement, and the achieved precision is \pm 0.05 and $\pm 0.01 \mathrm{~mm}$ for the size and alignment, respectively. The momentum distributions of the elastically scattered protons were used not only for monitoring the relative geometrical settings of the target, but also for the exact positions of the dipole field and the drift chambers [27].

As the next step of the analysis, the missing-mass spectra were determined in order (i) to distinguish between the signal and background, (ii) to evaluate the absolute beam momenta, and finally (iii) to extract the width of the $\eta^{\prime}$ meson. The spectra for the highest and lowest excess energies are shown in Fig. 2. It is important to stress that the background distribution is smooth in the whole range studied and that the signal from $\eta^{\prime}$ meson production shows up clearly. The figure illustrates that the spectrum at one energy can be used as a good estimate of the background to the spectra at the other energies. The method for the background subtraction is based on the observation that the shape of the multipion mass distribution does not change when the excess energy for the $p p \rightarrow p p \eta^{\prime}$ reaction varies by a few $\mathrm{MeV}$, which is small compared to the total available energy of about $500 \mathrm{MeV}$ [16]. The systematic error in the changes of the shape due to the method applied was estimated to be less than $1 \%$ even for shifts many times larger than the energy range relevant in these measurements [29]. In order to decrease the influence of the statistical fluctuation, the background for a given energy was taken from a second-order polynomial fit to the 
data at a different energy, which was shifted and normalized to the data of interest [27].

Owing to the large statistics of the momentum distributions for elastically scattered protons, relative differences between excess energies were determined with a negligible statistical error from the sizes of the kinematic ellipses [27]. Next, the absolute values of the excess energies were derived by comparing the position of the mean of the missing-mass peak for data closest to the threshold with the empirical value of the mass of the $\eta^{\prime}$ meson [4]. The "true" values of the beam momenta thus determined are $3210.7,3212.6,3213.5,3217.2$, and $3223.4 \mathrm{MeV} / \mathrm{c}$, corresponding to excess energies of $0.8,1.4,1.7,2.8$, and 4.8 MeV, respectively. The accuracy of the beam momentum determination amounts to $\pm 0.2 \mathrm{MeV} / c$ and is predominantly due to the uncertainty of the $\eta^{\prime}$ mass $(957.78 \pm 0.06) \mathrm{MeV} / c^{2}$ [4]. The systematically lower values of the true beam momenta of about $0.5 \mathrm{MeV} / c$ are consistent with previous experience at COSY where the real beam momentum was always smaller than the nominal value [30].

In order to derive the value of the $\eta^{\prime}$ width, the experimental missing-mass spectra were compared with distributions simulated with different values of $\Gamma_{\eta^{\prime}}$. In the simulations based on the GEANT3 packages [31], the response of the COSY-11 detector system to the $p p \rightarrow p p \eta^{\prime}$ reaction was generated, taking into account the geometry and material composition as well as relevant resolutions of the COSY-11 detector components, including the size of the target stream, the spatial and momentum spread of the beam, and also all known physical processes such as multiple scattering and nuclear reactions. In the simulations of the mass distribution, a Breit-Wigner formula for the $\eta^{\prime}$ meson was used. Afterwards, the generated events were analyzed in the same way as the experimental data, and sets of missing-mass spectra were reconstructed for the values of $\Gamma_{\eta^{\prime}}$ ranging from 0.14 to $0.38 \mathrm{MeV} / c^{2}$. Finally, the sum of the experimental background and the Monte Carlo missing-mass spectra for the $p p \rightarrow p p \eta^{\prime}$ reaction was fitted to the experimental data. The normalization factor of the $\eta^{\prime}$ signal was the only free parameter in this fit. The result of the fit is shown by solid lines in

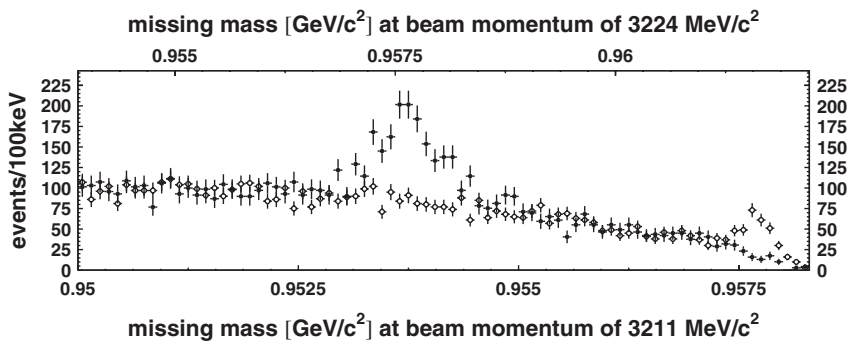

FIG. 2. Missing-mass spectra for the $p p \rightarrow p p X$ reaction determined at beam momenta of 3211 (open points) and $3224 \mathrm{MeV} / c$ (filled points). The filled points were shifted to the kinematic limit and normalized to the open points.
Fig. 3. The decrease of the width of the missing mass with decreasing excess energy is a kinematical effect reflecting the propagation of errors of momenta involved in the missing-mass calculations [32]. The simulations reproduce very well the change of the signal width with excess energy and thus validate the correctness of the established detector and target characteristics. The lower-right panel of Fig. 3 presents the dependence of the $\chi^{2}$ on the $\Gamma_{\eta^{\prime}}$ value. The minimum of $\chi^{2}$ is at $\Gamma_{\eta^{\prime}}=0.226 \mathrm{MeV} / c^{2}$, and the $1 \sigma$ statistical error is equal to $\pm 0.017 \mathrm{MeV} / c^{2}$.

The systematic error was estimated by studying the sensitivity of the result to the variation of parameters describing the experimental conditions in the analysis and in the simulation [27]. The contributions to the systematic error are (i) the target position $\left( \pm 0.006 \mathrm{MeV} / c^{2}\right)$ and size $\left( \pm 0.002 \mathrm{MeV} / c^{2}\right)$, (ii) the position and orientation of
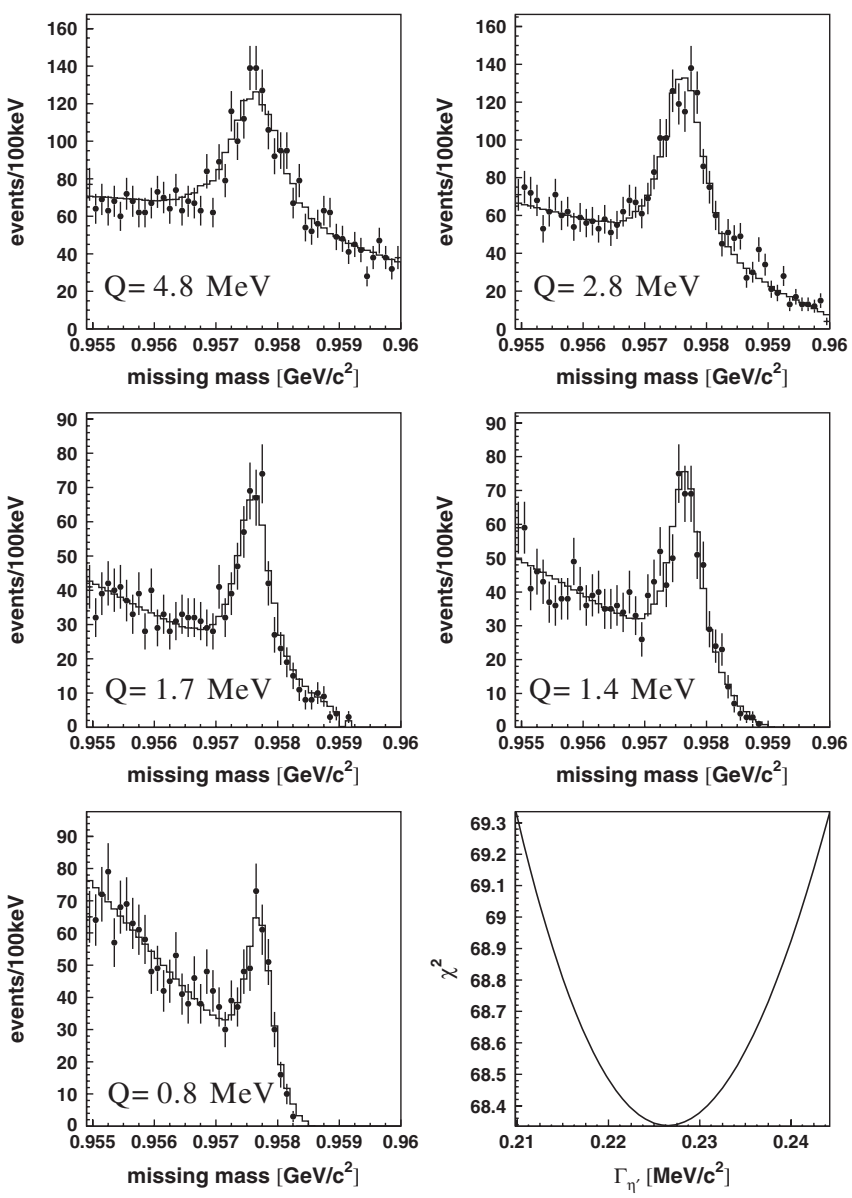

FIG. 3. The missing-mass spectra for the $p p \rightarrow p p X$ reaction. The $\eta^{\prime}$ meson signal is clearly visible. The experimental data are presented as points, while in each plot the line corresponds to the sum of the Monte Carlo generated signal for the $p p \rightarrow p p \eta^{\prime}$ reaction with $\Gamma_{\eta^{\prime}}=0.226 \mathrm{MeV} / c^{2}$ and the background obtained from another energy. The plot at the bottom right of the figure presents $\chi^{2}$ as a function of the $\Gamma_{\eta^{\prime}}$. The minimum value of the $\chi^{2}$ divided by the number of degrees of freedom amounts to 0.96 . 


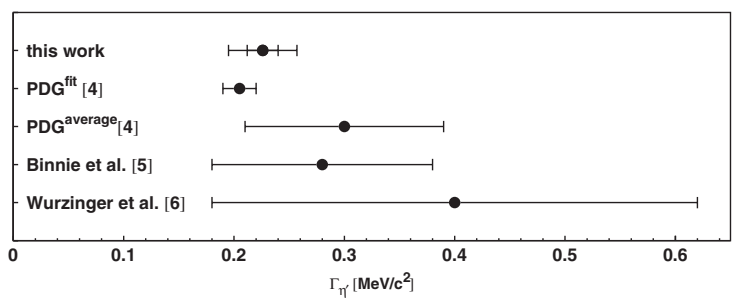

FIG. 4. Comparison of available values of $\Gamma_{\eta^{\prime}}$.

the drift chambers $\left( \pm 0.001 \mathrm{MeV} / c^{2}\right)$, (iii) the map of the magnetic field $\left( \pm 0.007 \mathrm{MeV} / c^{2}\right)$, and (iv) the absolute beam momentum determination $\left( \pm 0.003 \mathrm{MeV} / c^{2}\right)$. These values were estimated as the difference between the derived result of the $\Gamma_{\eta^{\prime}}$ and the $\Gamma_{\eta^{\prime}}$ values established by changing in the analysis and simulations a particular parameter by its error. The systematic error due to the method of the background subtraction $\left( \pm 0.006 \mathrm{MeV} / c^{2}\right)$ was established as the maximum difference between $\Gamma_{\eta^{\prime}}$ values determined when using experimental background shapes from different energies. The uncertainty due to the bin width $\left( \pm 0.004 \mathrm{MeV} / c^{2}\right)$ was estimated by changing the width of bins in the range from 0.1 to $0.04 \mathrm{MeV} / c^{2}$. Furthermore, the sensitivity of the result to the range of the missing-mass values used for the fit $\left( \pm 0.005 \mathrm{MeV} / c^{2}\right)$ was estimated by enlarging the mass range by seven bins on each side of the peak. The inaccuracy due to the model applied in the simulations for the proton-proton final state interaction $\left( \pm 0.003 \mathrm{MeV} / c^{2}\right)$ was estimated conservatively as a differences in results determined when using parameterization of the proton-proton $S$-wave interaction $[33,34]$ and when neglecting the final-state interactions. Finally, the total systematic error was estimated as the quadratic sum of the nine independent contributions mentioned above and is $0.014 \mathrm{MeV} / c^{2}$. Our final result is compared with earlier width determinations in Fig. 4.

In summary, the mass distribution of the $\eta^{\prime}$ meson has been measured with an experimental resolution of FWHM $=0.33 \mathrm{MeV} / c^{2}$. The $\eta^{\prime}$ meson was created in the $p p \rightarrow p p \eta^{\prime}$ reaction close to the kinematic threshold by using the low-emittance proton beam of the cooler synchrotron COSY incident on a stream of hydrogen clusters. The outgoing protons were detected by using the COSY-11 facility. The total width of the $\eta^{\prime}$ meson was extracted from the missing-mass spectra and amounts to $\Gamma_{\eta^{\prime}}=0.226 \pm$ 0.017 (stat) \pm 0.014 (syst) $\mathrm{MeV} / c^{2}$. The result does not depend on knowing any of the branching ratios or partial decay widths. The extracted $\Gamma_{\eta^{\prime}}$ value is in agreement with both previous direct determinations of this value $\left(\Gamma_{\eta^{\prime}}=\right.$ $0.28 \pm 0.10 \mathrm{MeV} / c^{2}[5]$ and $\Gamma_{\eta^{\prime}}=0.40 \pm 0.22 \mathrm{MeV} / c^{2}$ [6]). The achieved accuracy is similar to that obtained by the PDG from a fit to 51 measurements of branching ratios and cross sections $\left(\Gamma_{\eta^{\prime}}=0.204 \pm 0.015 \mathrm{MeV} / c^{2}\right)$ [4].

Finally, it is worth noting that the achieved mass resolution is of the same order as the total width of the $\eta^{\prime}$ meson itself, thereby excluding the possibility of a substructure in the $\eta^{\prime}$ signal at this level.

We are thankful to Professor Colin Wilkin for his v aluable comments and suggestions of improvements of this manuscript. The work was partially supported by the European Commission through the Research Infrastructures action of the Capacities Program. Call: FP7-INFRASTRUCTURES-2008-1, Grant Agreement No. 227431, by the PrimeNet, by the Polish Ministry of Science and Higher Education through Grants No. 1253/B/ H03/2009/36 and No. 1202/DFG/2007/03, by the German Research Foundation (DFG), by the FFE grants from the Research Center Jülich, and by the virtual institute Spin and strong $Q C D$ (VH-VP-231).

*eryk.czerwinski@lnf.infn.it

[1] R. Maier et al., Nucl. Instrum. Methods Phys. Res., Sect. A 390, 1 (1997).

[2] S. Brauksiepe et al., Nucl. Instrum. Methods Phys. Res., Sect. A 376, 397 (1996).

[3] P. Moskal et al., Phys. Rev. C 69, 025203 (2004).

[4] C. Amsler et al., Phys. Lett. B 667, 1 (2008).

[5] D. M. Binnie et al., Phys. Lett. B 83, 141 (1979).

[6] R. Wurzinger et al., Phys. Lett. B 374, 283 (1996).

[7] B. Di Micco, Acta Phys. Pol. B, Proc. Suppl. 2, 63 (2009).

[8] F. Ambrosino et al., J. High Energy Phys. 07 (2009) 105.

[9] M. Acciarri et al., Phys. Lett. B 418, 399 (1998).

[10] K. Karch et al., Z. Phys. C 54, 33 (1992).

[11] D. Williams et al., Phys. Rev. D 38, 1365 (1988).

[12] B. Borasoy et al., Phys. Lett. B 643, 41 (2006).

[13] A. Kupść et al., in Proceedings of MENU07 Conference, Julich, 2007, econf C070910, 256 (2007).

[14] B. Borasoy et al., AIP Conf. Proc. 950, 180 (2007).

[15] R. Nissler et al., AIP Conf. Proc. 950, 188 (2007).

[16] P. Moskal et al., Phys. Rev. Lett. 80, 3202 (1998).

[17] F. Hibou et al., Phys. Lett. B 438, 41 (1998).

[18] F. Balestra et al., Phys. Lett. B 491, 29 (2000).

[19] P. Moskal et al., Phys. Lett. B 474, 416 (2000).

[20] A. Khoukaz et al., Eur. Phys. J. A 20, 345 (2004).

[21] P. Klaja et al., Phys. Lett. B 684, 11 (2010).

[22] M. Aguilar-Benitez et al., Phys. Rev. Lett. 25, 1635 (1970).

[23] T. A. Lasinski et al., Rev. Mod. Phys. 45, S1 (1973).

[24] S. D. Bass, Phys. Lett. B 463, 286 (1999).

[25] D. Prasuhn et al., Nucl. Instrum. Methods Phys. Res., Sect. A 441, 167 (2000).

[26] H. Dombrowski et al., Nucl. Phys. A626, 427c (1997).

[27] E. Czerwiński, Ph.D. dissertation, Jagiellonian University, 2009; arXiv:0909.2781.

[28] P. Moskal et al., Nucl. Instrum. Methods Phys. Res., Sect. A 466, 448 (2001).

[29] P. Moskal et al., J. Phys. G 32, 629 (2006).

[30] J. Smyrski, AIP Conf. Proc. 950, 146 (2007).

[31] CERN Program Library Long Writeup W5013, 1994 (unpublished).

[32] E. Czerwiński, Diploma thesis, Jagiellonian University, 2006.

[33] J. Naisse et al., Nucl. Phys. A278, 506 (1977).

[34] P. Moskal et al., Phys. Lett. B 482, 356 (2000). 